\title{
Ag-plasma modification enhances bone apposition around titanium dental implants: an animal study in Labrador dogs
}

This article was published in the following Dove Press journal:

International Journal of Nanomedicine

14 January 2015

Number of times this article has been viewed

\author{
Shichong Qiao',* \\ Huiliang $\mathrm{Cao}^{2, *}$ \\ Xu Zhaol,* \\ Hueiwen Lo' \\ Longfei Zhuang' \\ Yingxin $\mathrm{Gu}^{\prime}$ \\ Junyu Shi' \\ Xuanyong Liu ${ }^{2}$ \\ Hongchang Lai' \\ 'Department of Oral and \\ Maxillofacial Implantology, Shanghai \\ Key Laboratory of Stomatology, \\ Shanghai Ninth People's Hospital, \\ School of Medicine, Shanghai Jiao Tong \\ University, ${ }^{2}$ State Key Laboratory \\ of High Performance Ceramics and \\ Superfine Microstructure, Shanghai \\ Institute of Ceramics, Chinese \\ Academy of Sciences, Shanghai, \\ People's Republic of China \\ *These authors contributed equally \\ to this work
}

Correspondence: Hongchang Lai Department of Oral and Maxillofacial Implantology, Shanghai Ninth People's Hospital, School of Medicine, Shanghai Jiao Tong University, 639 Zhizaoju

Road, Shanghai 2000 II, People's

Republic of China

Tel +86212327 I699 ext 5298

Fax +86 2I 53073068

Email lhc9@hotmail.com

Xuanyong Liu

Shanghai Institute of Ceramics, Chinese Academy of Sciences, No 1295 Ding-xi Road, Shanghai 200050, People's Republic of China

Tel +86 2I 524I 2409

Fax +86 21 5241 2409

Email xyliu@mail.sic.ac.cn
Abstract: Dental implants with proper antibacterial ability as well as ideal osseointegration are being actively pursued. The antimicrobial ability of titanium implants can be significantly enhanced via modification with silver nanoparticles (Ag NPs). However, the high mobility of $\mathrm{Ag}$ NPs results in their potential cytotoxicity. The silver plasma immersion ion-implantation (Ag-PIII) technique may remedy the defect. Accordingly, Ag-PIII technique was employed in this study in an attempt to reduce the mobility of Ag NPs and enhance osseointegration of sandblasted and acid-etched (SLA) dental implants. Briefly, 48 dental implants, divided equally into one control and three test groups (further treated by Ag-PIII technique with three different implantation parameters), were inserted in the mandibles of six Labrador dogs. Scanning electron microscopy, X-ray photoelectron spectroscopy, and inductively coupled plasma optical emission spectrometry were used to investigate the surface topography, chemical states, and silver release of SLA- and Ag-PIII-treated titanium dental implants. The implant stability quotient examination, Microcomputed tomography evaluation, histological observations, and histomorphometric analysis were performed to assess the osseointegration effect in vivo. The results demonstrated that normal soft tissue healing around dental implants was observed in all the groups, whereas the implant stability quotient values in Ag-PIII groups were higher than that in the SLA group. In addition, all the Ag-PIII groups, compared to the SLA-group, exhibited enhanced new bone formation, bone mineral density, and trabecular pattern. With regard to osteogenic indicators, the implants treated with Ag-PIII for 30 minutes and 60 minutes, with the diameter of the Ag NPs ranging from 5-25 nm, were better than those treated with Ag-PIII for 90 minutes, with the Ag NPs diameter out of that range. These results suggest that Ag-PIII technique can reduce the mobility of Ag NPs and enhance the osseointegration of SLA surfaces and have the potential for future use.

Keywords: surface modification, micro/nanostructure, silver, ion implantation, osseointegration

\section{Introduction}

Silver, a nonspecific biocidal agent, can act strongly against a broad spectrum of bacterial and fungal species, even antibiotic resistant strains. ${ }^{1,2}$ Silver nanoparticles (Ag NPs) are believed to be more reactive than the bulk metallic forms due to the larger active surface area. There is abundant research focusing on the antibacterial activity of Ag NPs..$^{3-5}$ Inhibition of bacterial growth was observed for both Gram-positive and Gram-negative bacteria, ${ }^{5}$ and no resistance has been convincingly demonstrated for clinically-relevant pathogens. ${ }^{4}$ Moreover, the bactericidal properties of Ag NPs are superior to other silver compounds, ${ }^{6}$ and it has diverse applications in the form of wound dressings, medical device coatings, Ag NP-impregnated textile fabrics, etc. ${ }^{7-9}$ 
However, the ultra-small size and unique properties, such as high mobility of Ag NPs, also arouse concerns about their potential cytotoxicity. Several studies have reported that uptake of Ag NPs significantly induces necrosis or apoptosis in several cell types. ${ }^{10,11}$ It is evident that Ag NPs can be ionized after entering the cells, and induce a series of proinflammatory responses, markedly increasing the expression of TNF- $\alpha .^{4,12}$ They have also been shown to cause cell death in vitro, including macrophage cells, liver cells, and neuronal cells. ${ }^{13-15}$ In addition, liver damage and decrease in several red blood cell parameters induced by Ag NPs were confirmed in vivo. ${ }^{16}$ Therefore, the key to reducing the toxic effect on mammalian cells is to restrict the uptake of $\mathrm{Ag}$ NPs. In order to limit silver uptake, Ag NPs were produced and embedded in titanium by a silver plasma immersion ionimplantation (Ag-PIII) process. We found the silver-plasma treated substrates, other than having good activity against bacteria, enhance the proliferation of the osteoblast-like cell line MG63. ${ }^{17}$ This finding provides a more hopeful direction in solving the cytotoxicity problem. However, the in vivo effect of silver-plasma modification on osseointegration is not well known.

Dental implants made of titanium and its alloys have been documented to have successful osseointegration. ${ }^{18-20}$ The combined treatment of sandblasted and acid-etched (SLA) titanium surfaces produces micropits, with sizes ranging from 0.5 to $2 \mu \mathrm{m}$ in diameter. ${ }^{21,22}$ This type of surface has been shown to actively stimulate bone cell differentiation and protein production in in vitro studies, ${ }^{23,24}$ while animal studies have demonstrated that implants with an SLA surface have large amounts of bone-to-implant contact (BIC), ${ }^{25,26}$ resulting in large removal torque values in functional testing of the bone contact. ${ }^{27,28}$ It is documented in clinical studies that these rough-surfaced dental implants have a 10-year success rate of over $90 \% .^{29-32}$ Besides the integration of implants with host bone, however, successful dental implant restorations should also have stable and healthy peri-implant soft and hard tissues. ${ }^{33}$ Peri-implant infections, including peri-implant mucositis and peri-implantitis, have become one of the most serious complications, which may cause the failure of dental implants. Many studies have shown a close association between the occurrence of implant loss and peri-implant infection. Bacterial contamination and the related risks of infection constitute the most serious complication. ${ }^{34,35}$ It is generally accepted that an important tactic to make implants antibacterial is to impregnate them with antibacterial agents. Therefore, a titanium dioxide tube surface was modified with a mixture of amoxicillin and poly-lactic-co-glycolic acid to obtain antibacterial properties. ${ }^{36}$ The antibacterial activity of sol-gel nitrogen oxide coatings on orthopedic implants was also investigated. ${ }^{37}$ However, most of the reports are addressed to verify efficacy against one or more bacterial strains but fall short of reporting its biocompatibility. As for the incorporation of Ag NPs, studies of the impact of Ag-PIII treatment on the implant osseointegration in vivo are also significantly insufficient.

Accordingly, we hypothesized that the Ag-PIII technique might minimize the mobility of Ag NPs embedded in the SLA surface, and this micro/nanostructured surface may possess better bioactive properties and further enhance implant osseointegration in vivo. Therefore, in this study, the SLA-treated titanium dental implants, in order to incorporate Ag NPs, were further modified by the Ag-PIII process. The surface properties were evaluated comprehensively. The bone apposition around the SLAand Ag-PIII-treated titanium dental implants was studied in Labrador dogs. To our knowledge, it is the first report to evaluate the combined action of Ag NPs and SLA titanium dental implant surfaces in vivo. We hope the results of this work will be a useful and significant reference for the potential properties of this implant modification and its future applications.

\section{Materials and methods Surface treatment characterization Ag-PIII}

Commercially pure titanium implants, which displayed a proprietary porous surface modification in the micrometer scale with a machined margin $(\varnothing 3.3 \mathrm{~mm}, 10 \mathrm{~mm}$ length, regular neck, standard, SLA surface; Trausim Medical Instrument Co. Ltd, Changzhou City, People's Republic of China), were chosen as SLA-based samples to be modified by the Ag-PIII technique. This implant system is being used in a clinical investigation of medical devices for human under the supervision of China Food and Drug Administration. The samples were transferred into the chamber of a Ag-PIII system, with a cathode rod made of pure silver metal, with a purity of $99.99 \%$ weight and diameter of $10 \mathrm{~mm}$. Then, the PIII technique was used to implant silver ions into the titanium dental implants surfaces, including the machined margin and the SLA-treated surface. The involved samples treated with various implantation parameters are listed in Table 1. The detailed Ag-PIII process was depicted in our previous study. ${ }^{17}$ The treated samples were used as three test groups. The implants without any treatment were used as a control group. 
Table I Details of the Ag-PIII parameters for the samples involved

\begin{tabular}{lll}
\hline Groups & Samples & Ag-PIII parameters \\
\hline Test group I & $30 \mathrm{~min}-30 \mathrm{Ag}$ PIII & Bias voltage $30 \mathrm{kV}$, duration $0.5 \mathrm{~h}$ \\
Test group 2 & 60 min-30 Ag PIII & Bias voltage $30 \mathrm{kV}$, duration I.0 h \\
Test group 3 & 90 min-30 Ag PIII & Bias voltage $30 \mathrm{kV}$, duration $1.5 \mathrm{~h}$ \\
Control group & SLA & N/A \\
\hline
\end{tabular}

Abbreviations: Ag-PIII, silver plasma immersion ion implantation; N/A, not available; SLA, titanium surfaces treated by sandblasting with large grit and acid etching procedure; 30 min-30 Ag PIII, titanium surfaces treated by first SLA procedure and then silver plasma immersion ion implantation at $30 \mathrm{kV}$ for 30 minutes; $60 \mathrm{~min}$ $30 \mathrm{Ag}$ PIII, titanium surfaces treated by first SLA procedure and then silver plasma immersion ion implantation at $30 \mathrm{kV}$ for 60 minutes; 90 min-30 Ag PIII, titanium surfaces treated by first SLA procedure and then silver plasma immersion ion implantation at $30 \mathrm{kV}$ for 90 minutes.

\section{Surface chemistry and structure characterization}

The rough surface morphology of the samples before and after Ag-PIII was examined by field emission scanning electron microscopy (JSM-6700F; JEOL, Tokyo, Japan). The chemical composition of the surfaces was determined by X-ray photoelectron spectroscopy (Kratos AXIS Ultra DLD). Three different areas were measured on three samples from each group.

\section{Silver release}

Each group of Ag-PIII-treated samples was incubated for 3 months in $10 \mathrm{~mL}$ water at $37^{\circ} \mathrm{C}$ without stirring. Inductively coupled plasma optical emission spectrometry (ICP) was used to determine the amounts of released silver as described previously. ${ }^{17}$

\section{Animal preparation}

Dogs, with the most similar bone structure to humans including both microstructure and macrostructure, are considered to be an ideal animal model for implant biomaterial research. ${ }^{38}$ Labrador dogs have been widely used for testing bone-implant materials. ${ }^{39-42}$ The aims of the present study were to explore the compatibility of Ag NPs-modified dental implants in the mandibles of Labrador dogs compared with the standard implants, and to answer the question, whether and how the physicochemical modification with Ag-PIII techniques affects the osseointegration in clinical and histological aspects.

Six adult (24-30 months old) male Labrador dogs, weighing $26.8-32.4 \mathrm{~kg}$, with fully erupted permanent dentition were used, following a protocol approved for this study by the Animal Care and Experiment Committee of Ninth People's Hospital, affiliated to Shanghai Jiao Tong University, School of Medicine. All the animals were obtained from the Laboratory Animal Center of Shanghai Jiao Tong University, maintained at the Animal Research Center of Shanghai Ninth People's Hospital, and had ad libitum access to water and soft-food diet throughout the study. The animals were routinely inspected and acclimatized in a 4-week period before initiation of the surgery. The Animal Research: Reporting of In Vivo Experiments guidelines for preclinical in vivo studies were followed. ${ }^{43,44}$

\section{Surgical procedure}

This study involved two surgical stages. During each surgical procedure, the animals were under general anesthesia. Ketamine (10 mg/kg intramuscular) was used as the basic general anesthesia agent, and procaine (procaine $40 \mathrm{mg}$, adrenaline $0.05 \mathrm{mg}$ ) was used for local anesthesia.

At the first stage, all the mandibular premolars and first molars were extracted bilaterally. Penicillin (800,000 U/day, 3 days) was given following the extraction procedures. The recovery period was maintained as 3 months, when the alveolar processes were fully healed.

During the second stage, a midcrestal incision was made in the premolar to molar region in the left side of the mandible. Full-thickness mucoperiosteal flaps were elevated labially and lingually to expose the alveolar ridge. Four experimental sites allocated to one control group and three test groups were identified with a $2 \mathrm{~mm}$ round bur afterwards. Therefore, six Labrador dogs were used in the study, and four implant sites, addressed as A, B, C, and D, were located from anterior to posterior on each side of the mandibular bones. Thus, in total, 48 implant sites were achieved, comprising 12 sites for each A, B, C, and D. We assigned three of each kind of site to every group of samples. The surgical preparation of the sites followed the manual of the implant system. Pilot drills (Ø $2.2 \mathrm{~mm})$ and twist drills (Ø $2.8 \mathrm{~mm})$ were used successively to prepare each experimental site for an implant $10 \mathrm{~mm}$ in length and $3.3 \mathrm{~mm}$ in diameter. Then, the thread tapper (Ø $3.3 \mathrm{~mm}$ ) was used to reduce the torque force during the implant insertion. Four kinds of implants were inserted into the four sites, and the margin (machined neck-fixture) was located at the level of the buccal margin of the socket wall. Titanium healing caps were attached to all implants. The surgical wound was carefully closed. All the implants were left to heal nonsubmerged. The surgical process was the same as above for the right side of the mandible.

After the surgical procedures, the animals received antibiotics (amoxicillin $500 \mathrm{mg}$, twice daily) and analgesics (ibuprofen $600 \mathrm{mg}$, three times a day) via the systemic route for 3 days. In addition, a plaque control program, cleaning of both the teeth and implants with toothbrush and dentifrice 
without anesthesia (once a day, 5 days a week), was introduced. It was terminated 4 weeks later.

\section{Implant stability assessment}

Implant stability quotient (ISQ) was measured by means of the resonance frequency analysis (Osstell ${ }^{\circledR}$; Integration Diagnostics, Savadaled, Sweden) immediately after implant placement (primary stability) and subsequently at weeks 4,8 , and 12 . For each single implant, the average value of six measurements performed directly over the fixation was calculated. The range of ISQ was from 1 to 100 , and a higher ISQ indicates increased stability. ISQ is the most reported clinical tool for evaluating dental implant stability and roughly reveals the condition of bone tissue formation around dental implants. The detailed ISQ information will be considered in the discussion section.

\section{Sample preparation}

At 12 weeks following the implant insertion procedure, all animals were sacrificed with an overdose of pentothal sodium under general anesthesia via injection of ketamine $(10 \mathrm{mg} / \mathrm{kg})$ and xylazine $(4 \mathrm{mg} / \mathrm{kg})$ intramuscularly, and were then perfused through carotid arteries with a fixative mixture of glutaraldehyde (5\%) and formaldehyde (4\%) buffered to $\mathrm{pH}$ 7.2. The mandibles were dissected and separated into two parts, the left and the right. All the healing caps were removed from the implants. Each sample with four groups of implants was fixed in 10\% neutral-buffered formalin solution for Microcomputed tomography (micro-CT) assay and histomorphometric observation.

\section{Micro-CT assay}

Structural analysis of the fixed samples was performed using micro-CT (GE eXplore Locus SP Micro-CT; USA) at $15 \mu \mathrm{m}$ resolution. The scanning parameters were set at $80 \mathrm{kV}$ voltage and $80 \mathrm{~mA}$ current, with an exposure time of 3,000 ms. To obtain parameters of bone volume (BV)/total volume fraction and bone mineral density (BMD) of the new bone formed around the implant, a region with a radius of $1 \mathrm{~mm}$ from implant surface was selected for analysis as reported previously. ${ }^{45}$ Trabecular thickness and trabecular number of new bone were analyzed as well.

\section{Histomorphometric observation}

After micro-CT assay, the 48 dental implant mandibular specimens were dehydrated using an ascending series of alcohols, from $75 \%$ to absolute ethanol, and then embedded in polymethylmethacrylate for undecalcified sectioning. The fully polymerized specimens were sectioned into $150 \mu \mathrm{m}$ thick sections using a Leica SP1600 saw microtome (Leica, Hamburg, Germany). All specimens were subsequently glued with acrylic cement to silanized glass sides, then ground and polished to a final thickness of about $30 \mu \mathrm{m}$. The specimens were finally stained with van Gieson's picro fuchsin for histological observation.

The histomorphometric measurements were performed by an investigator who was masked to the experimental conditions. Image-Pro Plus 6.0 (Media Cybernetics Inc., Silver Spring, MD, USA) was applied to evaluate the digital images captured using a light microscope (CX21: Olympus, Tokyo, Japan). The most central section from each specimen was selected for the analysis.

The following measurements were made:

1. The height of the suprabony connective tissue (aJE-B): from the level of the apical termination of the junctional epithelium (aJE) to the marginal level of BIC in buccal areas.

2. Bone density within the threads (BDWT): percentage of mineralized tissue area in the adjoining bone within the implant threads. The calculation formula is shown below:

$$
\text { BDWT }=\frac{\text { Bone area }}{\text { Bone area }+ \text { marrow spaces area }}
$$

3. Percentage of BIC: the ratio of the length of bone in direct contact with implant surface.

\section{Statistical analysis}

Each group encompassed 12 samples. The results of the measurements were presented as mean \pm standard deviation. Statistical comparisons were carried out via analysis of variance (ANOVA) and Student-Newman-Keuls test. All statistical analysis was carried out using SPSS (version 16.0; SPSS, USA). The differences were considered statistically significant when $* P<0.05$ and $* * P<0.01$.

\section{Results}

\section{Characterization of the Ag-PIII implants}

As shown in Figure 1, a microstructure evolution can be seen on the SLA surface after undergoing Ag-PIII at $30 \mathrm{kV}$ for 30 minutes, 60 minutes, and 90 minutes (designated as 30 min-30 Ag PIII, 60 min-30 Ag PIII, 90 min-30 Ag PIII, respectively). The original SLA implants have surfaces that are very rough but regular in appearance, which are covered with macropits about $10-40 \mu \mathrm{m}$ in diameter, and each macropit is covered with micropits $2-4 \mu \mathrm{m}$ in diameter (Figure 1A). Moreover, even smaller pits $<1 \mu \mathrm{m}$ in diameter can be found in these micropits. Although rough on the microscale, the SLA 

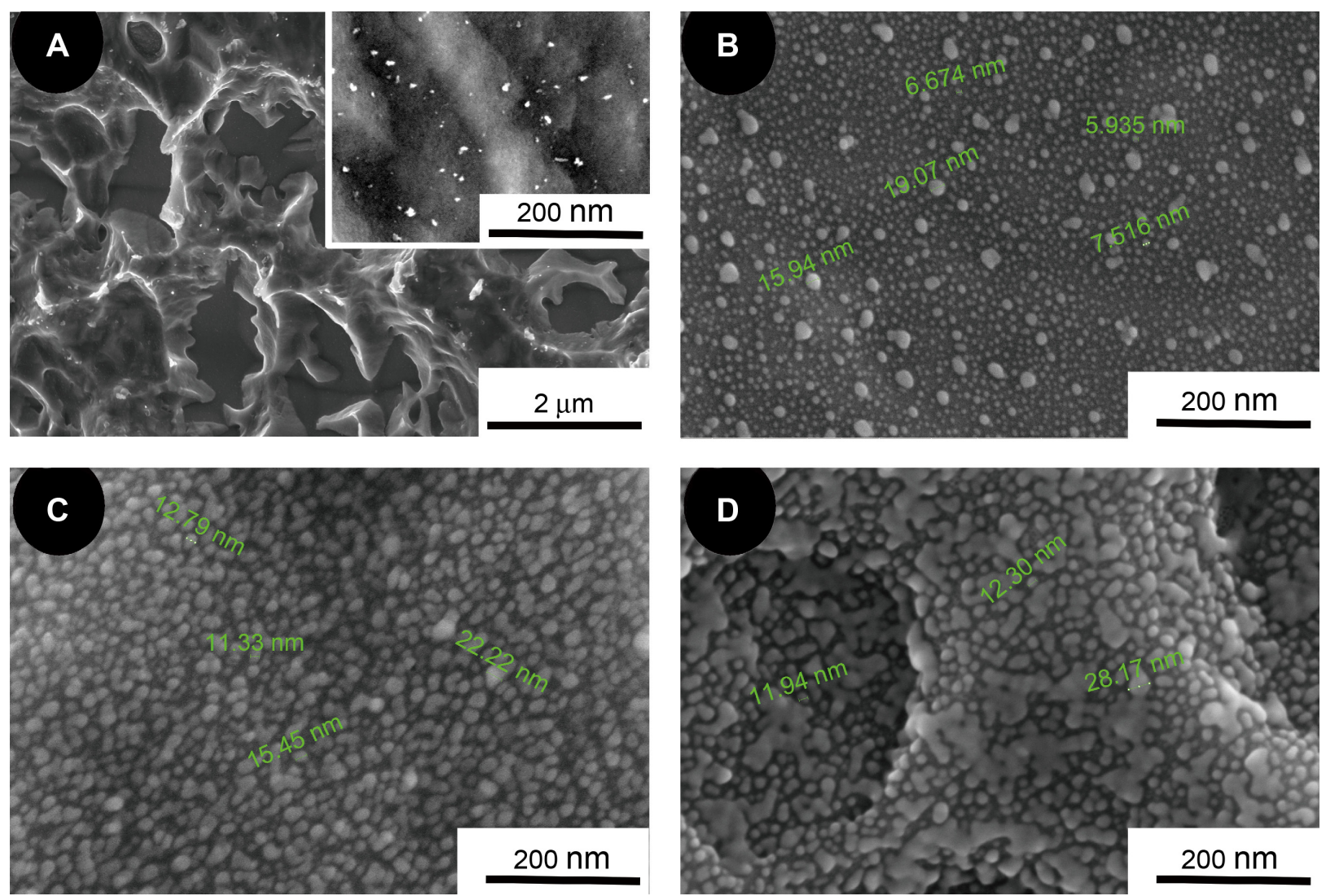

Figure I SEM photographs of (A) the untreated SLA surface and higher magnification image inserted, (B) 30 min-30 Ag PIII, (C) 60 min-30 Ag PIII, (D) 90 min-30 Ag PIII. Notes: (A) Particles around $15 \mathrm{~nm}$ scattered in particles around $5 \mathrm{~nm}$; (B) particles with sizes around I0-25 nm; (C) particles fused together. The peak of the particle size is greater than $40 \mathrm{~nm}$.

Abbreviations: Ag-PIII, silver plasma immersion ion implantation; SEM, scanning electron microscopy; SLA, titanium surfaces treated by sandblasting with large grit and acid etching procedure; 30 min-30 Ag PIII, titanium surfaces treated by first SLA procedure and then silver plasma immersion ion implantation at $30 \mathrm{kV}$ for 30 minutes; 60 min-30 $\mathrm{Ag}$ PIII, titanium surfaces treated by first SLA procedure and then silver plasma immersion ion implantation at $30 \mathrm{kV}$ for 60 minutes; 90 min-30 Ag PIII, titanium surfaces treated by first SLA procedure and then silver plasma immersion ion implantation at $30 \mathrm{kV}$ for 90 minutes.

surfaces are relatively smooth on the nanoscale to some extent (the insert in the upper-right corner of Figure 1A). However, after Ag-PIII, it becomes rough due to the emergence of nanoparticles. These particles, well-imposed in the microrough structure, can be detected from all kinds of Ag-PIII samples under high magnification (Figure 1B-D), thus forming a nano/ micron hierarchical structure. Although the microtopography of SLA-treated implants was retained after Ag-PIII, the nanotopography on the Ag-PIII implants are different. On $30 \mathrm{~min}-$ $30 \mathrm{Ag}$ PIII, particles with a mixture of two distinctive sizes are homogeneously distributed. Most of the particles are about 5 $\mathrm{nm}$ in scale, scattered with a small amount of particles around $15 \mathrm{~nm}$ (Figure 1B). With the increasing Ag-PIII time, the particles become larger and a significantly higher particle density can be observed. On 60 min-30 Ag PIII, particles with sizes around 10-25 nm were evenly distributed (Figure 1C), while on 90 min-30 Ag PIII, particles began to fuse together. The peak of the particle size is greater than $40 \mathrm{~nm}$ (Figure 1D), and the particles are not evenly distributed.
Although the particle size distributions are entirely different, the X-ray photoelectron spectroscopy investigation reveals similar chemical states of silver in these Ag-PIIItreated surfaces. The Ag 3d doublet two peaks located at $374.3 \mathrm{eV}(\mathrm{Ag} \mathrm{3d3/2})$ and $368.3 \mathrm{eV}$ (Ag 3d5/2) corresponds to metallic silver ${ }^{46}$ (Figures 2 and 3), demonstrating that the above mentioned particles on Ag-PIII samples are Ag NPs. In addition, the release of silver from the samples was evaluated by ICP. Less than a $10 \mathrm{ppb}$ silver (per square centimeter) can be detected after incubating the treated implants in pure water for over 90 days at $37^{\circ} \mathrm{C}$, signifying that the silver release was minimal.

\section{Clinical observation}

All the implants healed uneventfully in this study. No implants were lost through the follow-up period. The peri-implant soft tissues showed no clinical signs of overt inflammation. No significant complications were observed (Figure 4). 


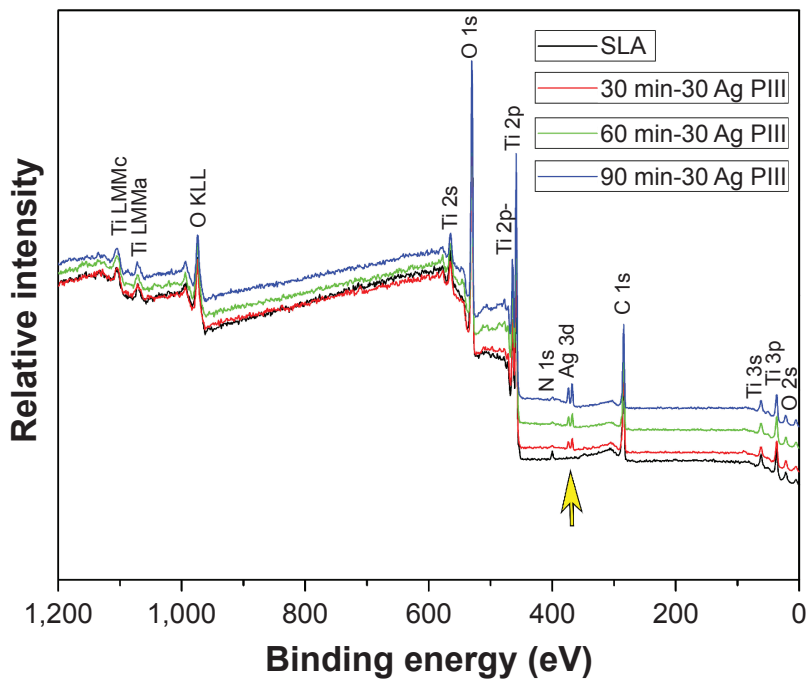

Figure 2 The typical XPS spectra of four different groups.

Notes: The yellow arrow indicates the Ag 3d doublet two peaks.

Abbreviations: Ag-PIII, silver plasma immersion ion implantation; SLA, titanium surfaces treated by sandblasting with large grit and acid etching procedure; XPS, X-ray photoelectron spectroscopy; 30 min-30 Ag PIII, titanium surfaces treated by first SLA procedure and then silver plasma immersion ion implantation at $30 \mathrm{kV}$ for 30 minutes; 60 min-30 Ag PIII, titanium surfaces treated by first SLA procedure and then silver plasma immersion ion implantation at $30 \mathrm{kV}$ for 60 minutes; 90 min$30 \mathrm{Ag}$ PIII, titanium surfaces treated by first SLA procedure and then silver plasma immersion ion implantation at $30 \mathrm{kV}$ for 90 minutes.

\section{Implant stability assessment}

At the time of implant placement, all implants had an ISQ over 65 , indicating that good primary stability had been achieved. During the first 4 weeks, the ISQ value decreased gradually to below 59. From then, the panel meter increased significantly to around 70 in the following 8 weeks. No implants showed at any time a clinical mobility. Remarkably, the outcomes of 8 weeks were significantly higher in Ag-PIII

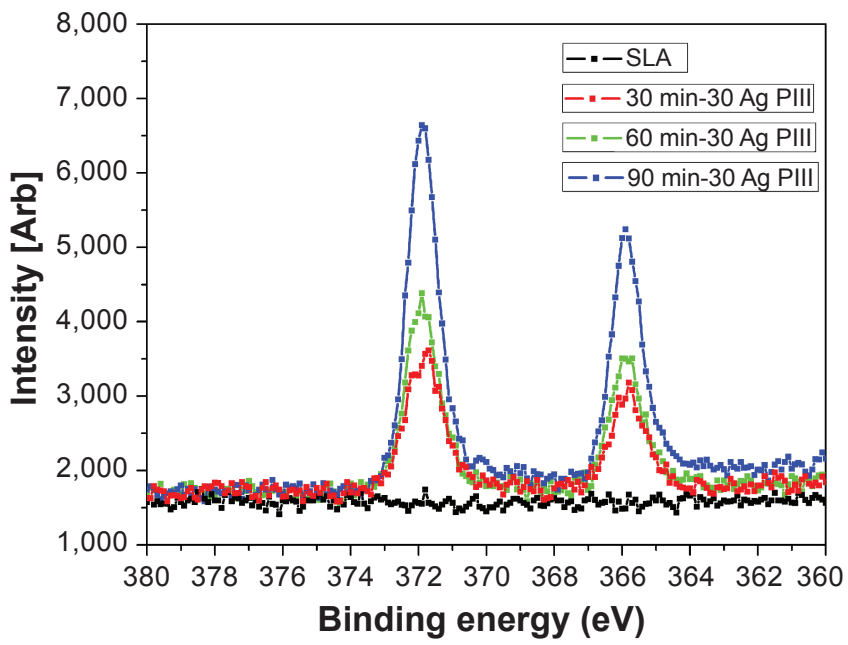

Figure 3 The relative $\mathrm{Ag} 3 \mathrm{~d}$ XPS spectrum.

Notes: The peaks can be indexed to the typical binding energies for $\mathrm{Ag} 3 \mathrm{~d} 3 / 2$ and $\mathrm{Ag} 3 \mathrm{~d} 5 / 2$ in $\mathrm{TiO} 2$, respectively.

Abbreviations: Ag-PIII, silver plasma immersion ion implantation; SLA, titanium surfaces treated by sandblasting with large grit and acid etching procedure; $\mathrm{TiO} 2$, titanium dioxide; XPS, X-ray photoelectron spectroscopy; 30 min-30 Ag PIII, titanium surfaces treated by first SLA procedure and then silver plasma immersion ion implantation at $30 \mathrm{kV}$ for 30 minutes; 60 min-30 Ag PIII, titanium surfaces treated by first SLA procedure and then silver plasma immersion ion implantation at $30 \mathrm{kV}$ for 60 minutes; 90 min-30 Ag PIII, titanium surfaces treated by first SLA procedure and then silver plasma immersion ion implantation at $30 \mathrm{kV}$ for 90 minutes.

groups than the control $(P<0.01)$. However, the difference narrowed for the 12 weeks result.

\section{Micro-CT evaluation of bone formation}

Reconstructed micro-CT images of transverse sections along the central axis of dental implants are shown in Figure 5A. The new bone had expanded and occupied almost the whole of the thread region in the Ag-PIII groups. Morphometric

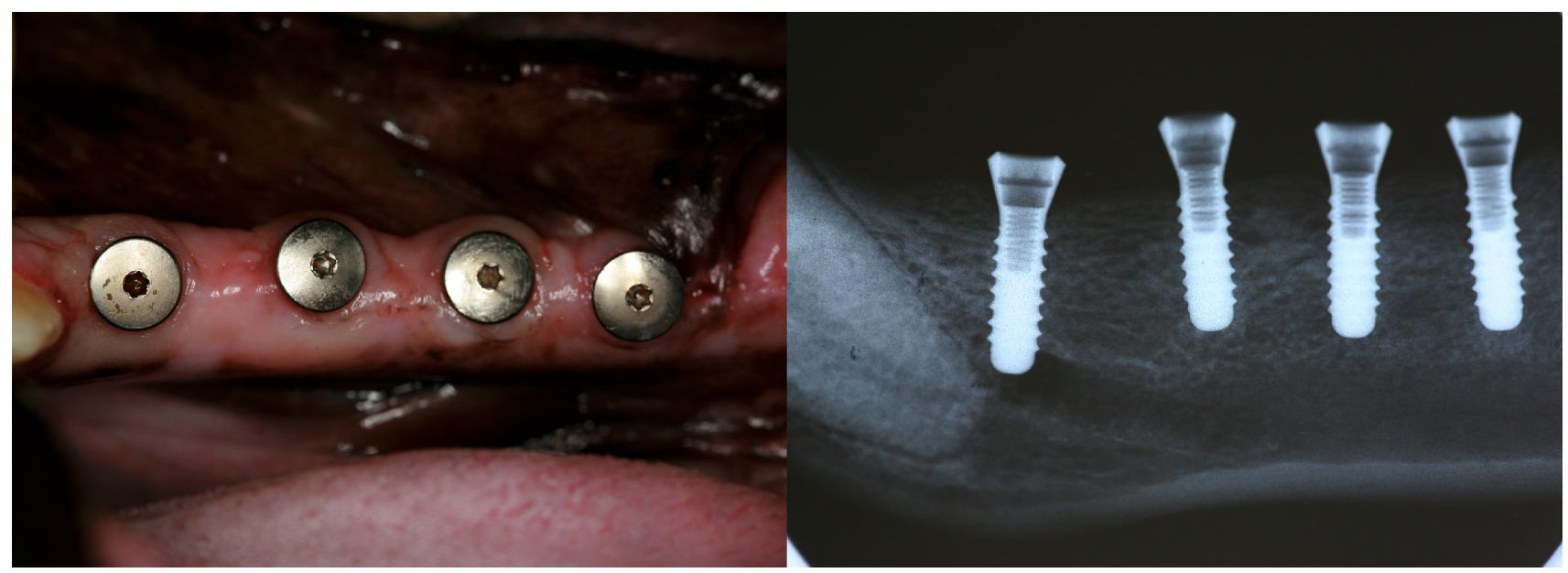

Figure 4 Clinical photograph and the corresponding radiograph at 12 weeks postsurgery.

Notes: The four implant sites healed unsubmerged. The peri-implant soft tissues showed no clinical signs of overt inflammation. The radiograph picture showed good osseointegration. 


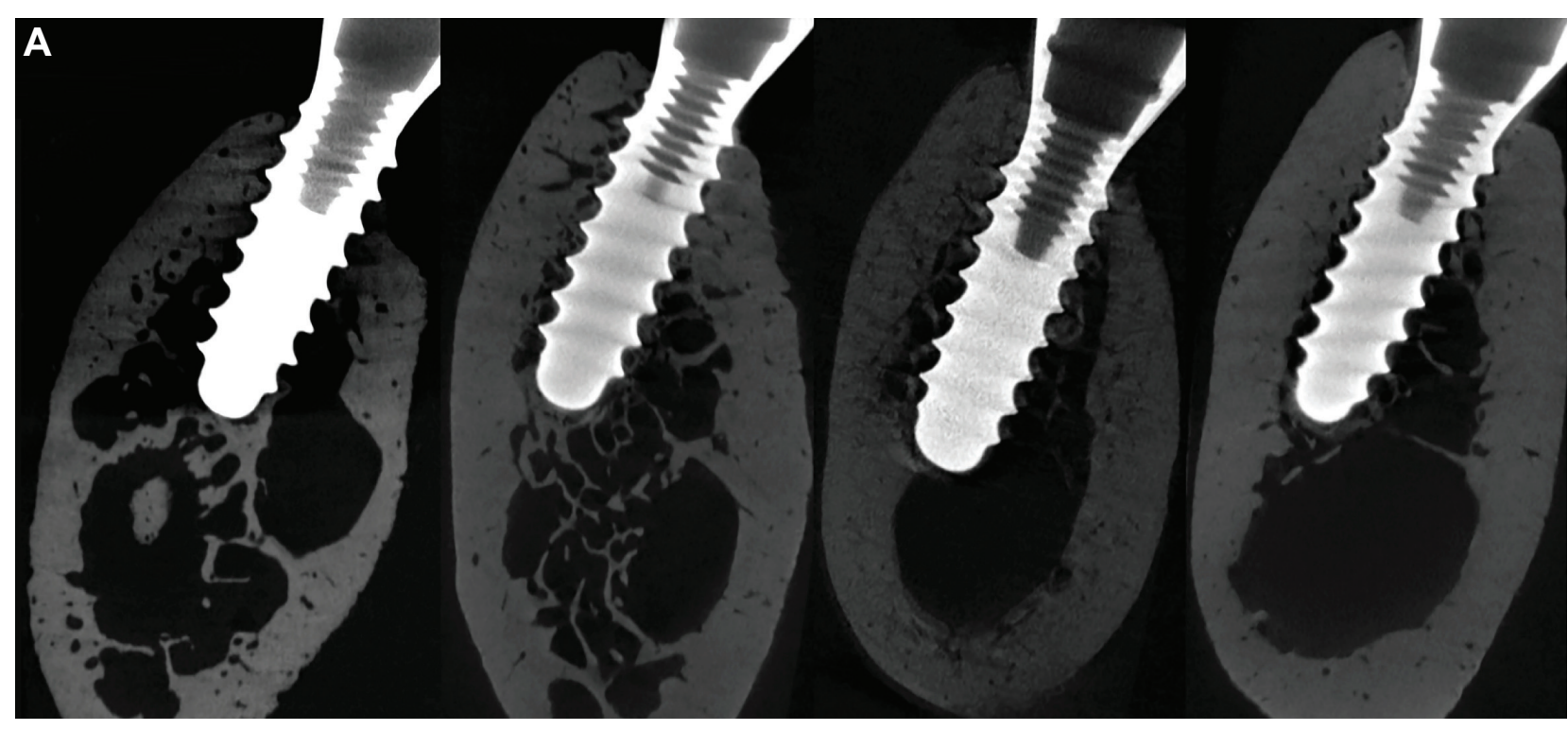

SLA

B
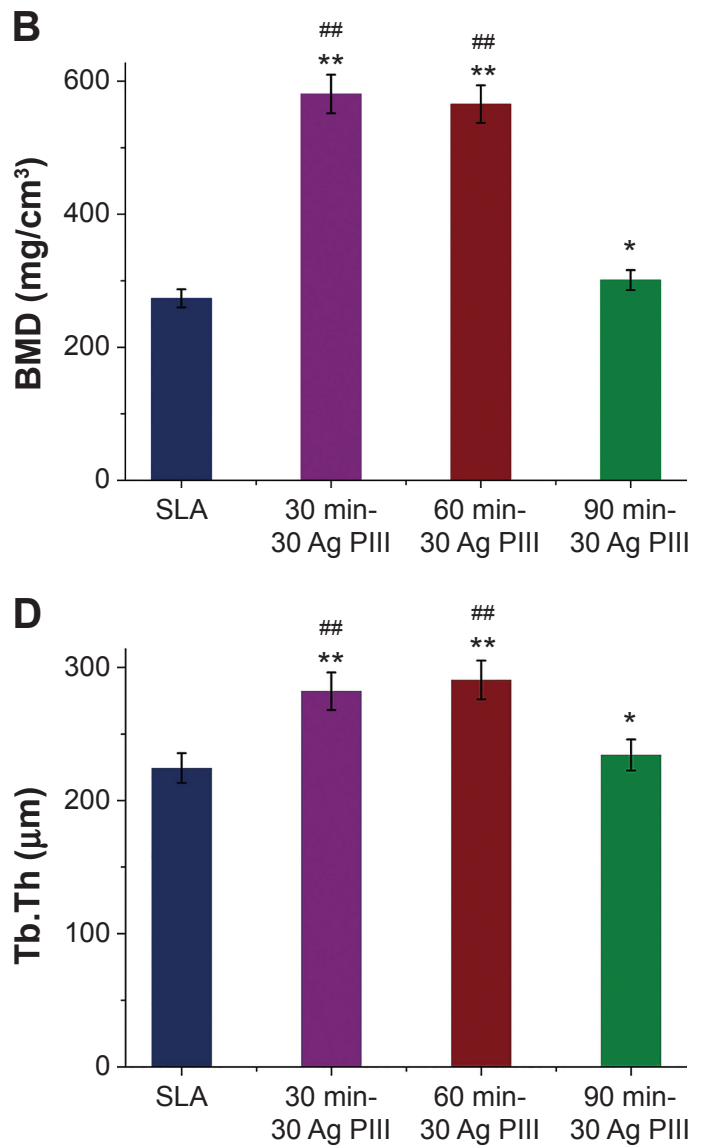

$60 \min -30$ Ag PIII

90 min-30 Ag PIII
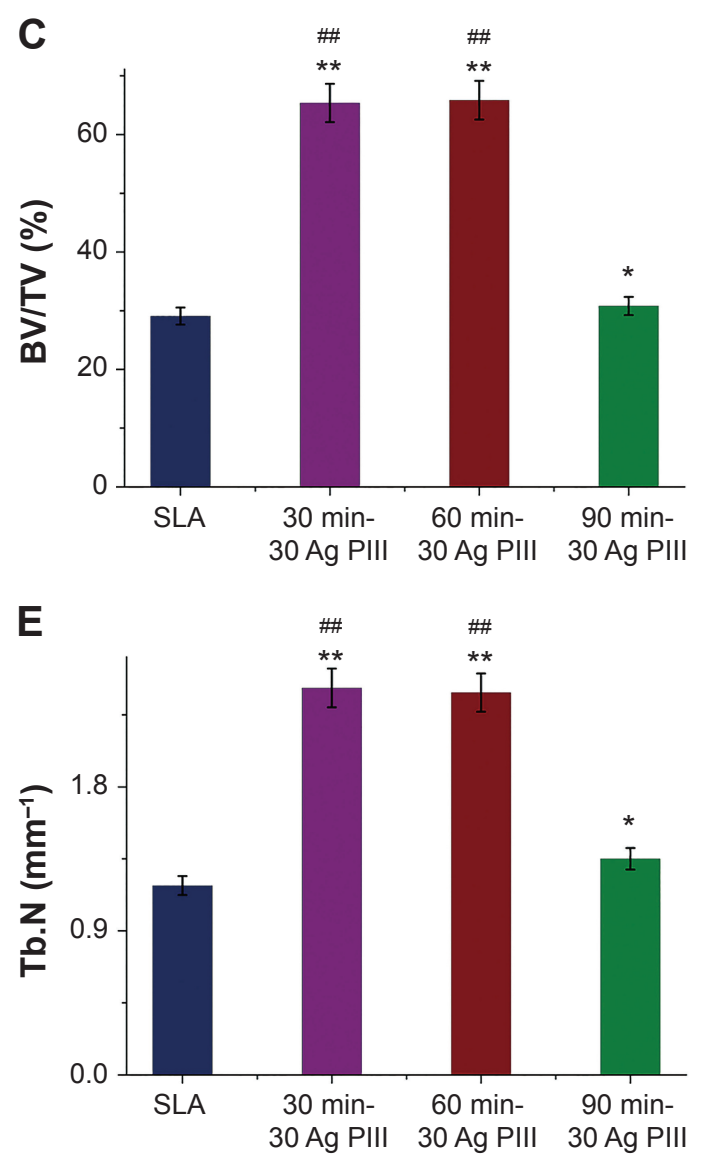

Figure 5 Radiography and micro-CT evaluation of the bone tissue around dental implants at 12 weeks after implantation.

Notes: Control group (SLA), 30 min-30 Ag PIII treated group, 60 min-30 Ag PIII treated group, 90 min-30 Ag PIII treated group (A-E from left to right). Representative photographs showed obviously more mineralized tissue formed in the Ag PIII groups than the control (A). The BMD, BV/TV, Tb.Th, Tb.N of the bone volume adjacent to the implant surface were quantified (B-E respectively); ${ }^{*} P<0.01$ versus the control SLA group; $* * P<0.05$ versus the control SLA group; ${ }^{\#} P<0.01$ versus the 90 min$30 \mathrm{Ag}$ PIII group.

Abbreviations: Ag-PIII, silver plasma immersion ion implantation; BMD, bone mineral density; BV/TV, bone volume/total volume; CT, computed tomography; SLA, titanium surfaces treated by sandblasting with large grit and acid etching procedure; Tb.N, trabecular number; Tb.Th, trabecular thickness; 30 min-30 Ag PIII, titanium surfaces treated by first SLA procedure and then silver plasma immersion ion implantation at $30 \mathrm{kV}$ for 30 minutes; 60 min-30 Ag PIII, titanium surfaces treated by first SLA procedure and then silver plasma immersion ion implantation at $30 \mathrm{kV}$ for 60 minutes; 90 min-30 Ag PIII, titanium surfaces treated by first SLA procedure and then silver plasma immersion ion implantation at $30 \mathrm{kV}$ for 90 minutes. 
measurements were performed to analyze the newly formed bone quantitatively. BV around the implant surface of 30 min-30 Ag PIII and 60 min-30 Ag PIII were significantly higher than that of the other two groups $(P<0.01)$. In addition, it was statistically higher in the 90 min-30 Ag PIII group than the control $(P<0.05)$, but not significantly $(P>0.01)$. No statistical difference could be found between the $30 \mathrm{~min}-30$ Ag PIII and 60 min-30 Ag PIII groups. Similar results were obtained for BMD, BV/total volume, trabecular thickness, and trabecular number (Figure 5B-E, respectively).

\section{Histological analysis}

\section{Soft tissue around different implants}

A light microscope was used to observe and photograph the ground sections. Figure 6 shows the identified landmarks used for the linear measurements. These included 1) the most occlusal point of the peri-implant mucosa, 2) the level of the aJE, and 3) the marginal position of bone-implant contact. The distances between the landmarks were measured (Table 2). The epithelial structure terminated 2-2.5 mm apical of the soft tissue margin (peri-implant mucosa-aJE) and $1-1.5 \mathrm{~mm}$ from the bone crest (aJE-B). No statistical difference was found $(P>0.05)$. The connective tissue in

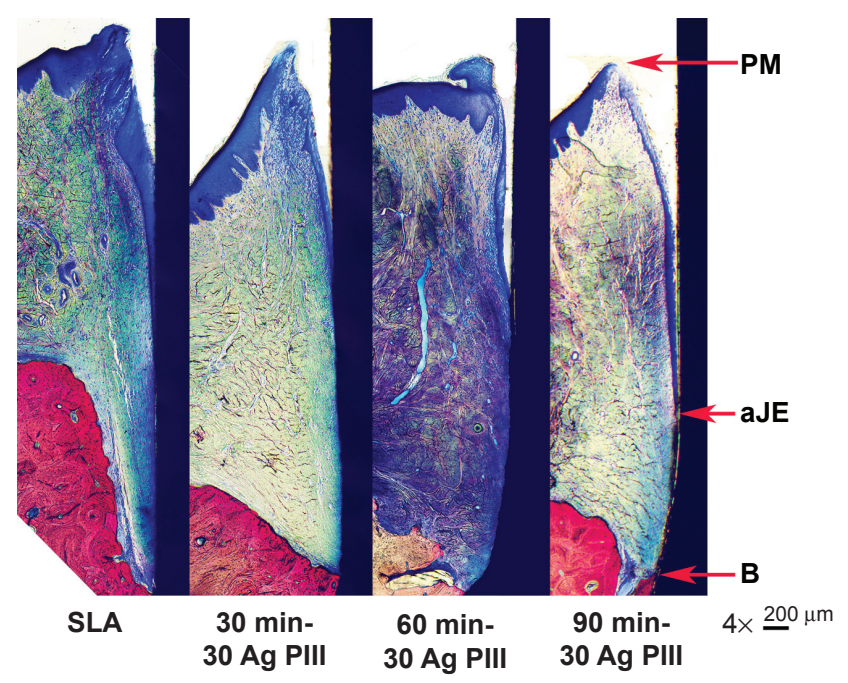

Figure 6 Histological pictures demonstrating the landmarks which were used for the assessment of linear distance for the soft tissue.

Notes: Control group (SLA), 30 min-30 Ag PIII treated group, 60 min-30 Ag PIII treated group, 90 min-30 Ag PIII treated group (from left to right). No significant difference was observed.

Abbreviations: Ag-PIII, silver plasma immersion ion implantation; aJE, apical termination of the junctional epithelium; B, marginal bone crest; PM, peri-implant mucosa margin; SLA, titanium surfaces treated by sandblasting with large grit and acid etching procedure; $30 \mathrm{~min}-30 \mathrm{Ag}$ PIII, titanium surfaces treated by first SLA procedure and then silver plasma immersion ion implantation at $30 \mathrm{kV}$ for 30 minutes; 60 min-30 Ag PIII, titanium surfaces treated by first SLA procedure and then silver plasma immersion ion implantation at $30 \mathrm{kV}$ for 60 minutes; 90 min$30 \mathrm{Ag}$ PIII, titanium surfaces treated by first SLA procedure and then silver plasma immersion ion implantation at $30 \mathrm{kV}$ for 90 minutes.
Table 2 Results from the soft tissue histometric measurements $(\mathrm{mm})$

\begin{tabular}{lll}
\hline & PM-aJE & aJE-B \\
\hline SLA & $2.48 \pm 0.57$ & $1.33 \pm 0.20$ \\
30 min-30 Ag PIII & $2.27 \pm 0.42$ & $1.34 \pm 0.43$ \\
60 min-30 Ag PIII & $2.27 \pm 0.43$ & $1.27 \pm 0.26$ \\
90 min-30 Ag PIII & $2.36 \pm 0.36$ & $1.28 \pm 0.23$ \\
\hline
\end{tabular}

Notes: PM-aJE, distance between PM and aJE; aJE-B, distance between aJE and B. No statistical differences can be found among the groups.

Abbreviations: Ag-PIII, silver plasma immersion ion implantation; aJE, apical termination of the junctional epithelium; B, marginal bone crest; PM, peri-implant mucosa margin; SLA, titanium surfaces treated by sandblasting with large grit and acid etching procedure; 30 min-30 Ag PIII, titanium surfaces treated by first SLA procedure and then silver plasma immersion ion implantation at $30 \mathrm{kV}$ for 30 minutes; 60 min-30 Ag PIII, titanium surfaces treated by first SLA procedure and then silver plasma immersion ion implantation at $30 \mathrm{kV}$ for 60 minutes; 90 min$30 \mathrm{Ag}$ PIII, titanium surfaces treated by first SLA procedure and then silver plasma immersion ion implantation at $30 \mathrm{kV}$ for 90 minutes.

the aJE-B region appeared to be in direct contact with the surface of the implant, which formed a good soft tissue seal to protect the bone tissue from substances in the oral cavity. Detailed characterization of the microstructure in soft tissue could not be observed.

\section{Bone tissue around different implants}

Figure 7A shows the histological sections stained with van Gieson's picro fuchsin. All implants showed bone formation directly onto the implant surfaces, which indicated histological osseointegration. Significantly, more spaces between the mineralized tissues and the implant surface were observed at the interface of bone and SLA samples while Ag-PIII modification promoted new bone formation around the implant, demonstrating enhanced osseointegration. Two histological evaluations, the percentage of BDWT and BIC ratio, were carried out. The histomorphometric analysis confirmed the micro-CT results. Both BDWT and BIC for the Ag-PIII groups were statistically higher than those detected with the SLA groups (Figure 7B and C). Remarkably, BIC for the 30 min-30 Ag PIII and 60 min-30 Ag PIII groups were $73.18 \pm 5.23$ and 69.92 \pm 4.10 , respectively, significantly higher than that of SLA and 90 min-30 Ag PIII groups (61.99 \pm 4.66 and 66.05 \pm 3.97 , respectively, $P<0.01$ ). This is similar to the differences observed in BDWT among the groups. The 30 min-30 Ag PIII and 60 min-30 Ag PIII groups demonstrated a greater BDWT of $77.58 \pm 4.39$ and $77.97 \pm 3.34$, respectively, significantly higher than those of the SLA $(66.52 \pm 3.46)$ and 90 min-30 Ag PIII groups (69.69 3.68$)(P<0.01)$.

\section{Discussion}

Various bioactive trace elements have been used to promote the osseointegration performance and introduce some positive 
A

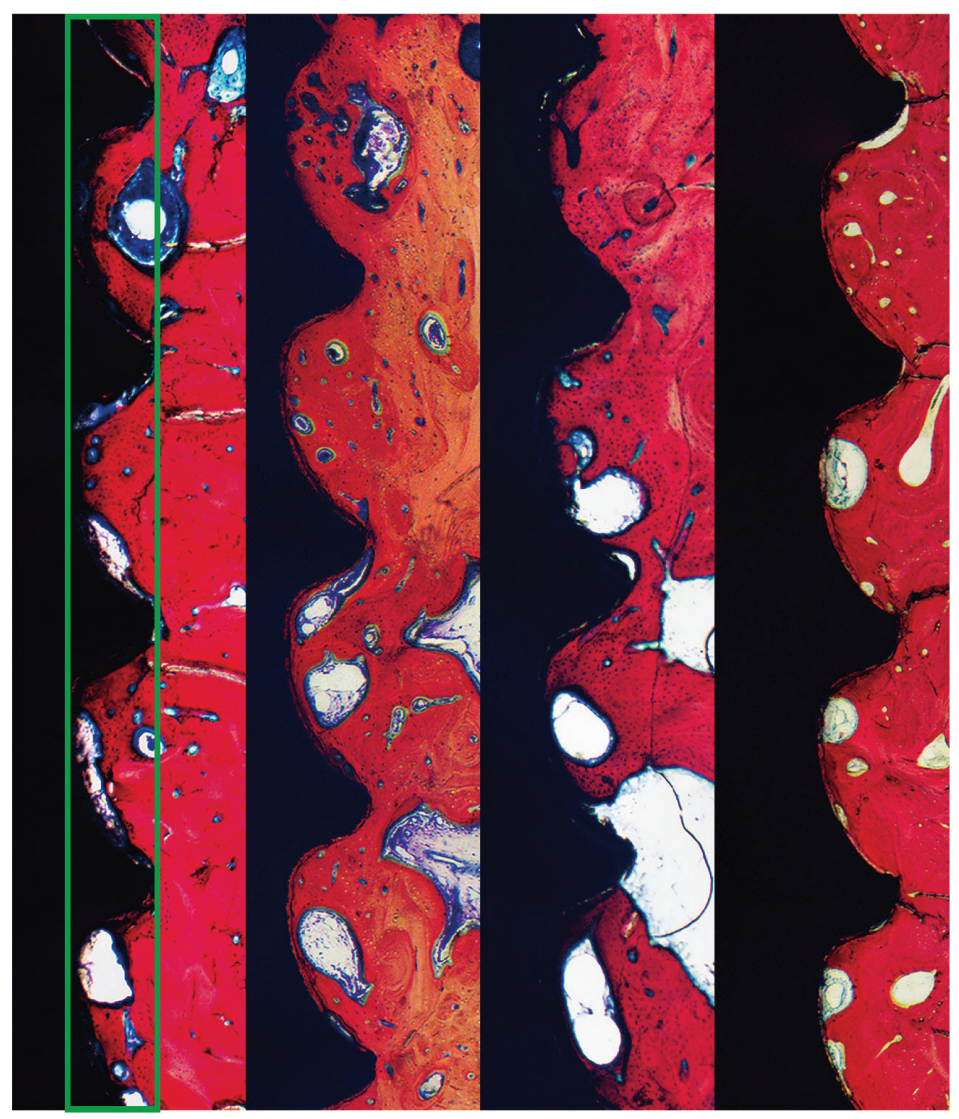

B

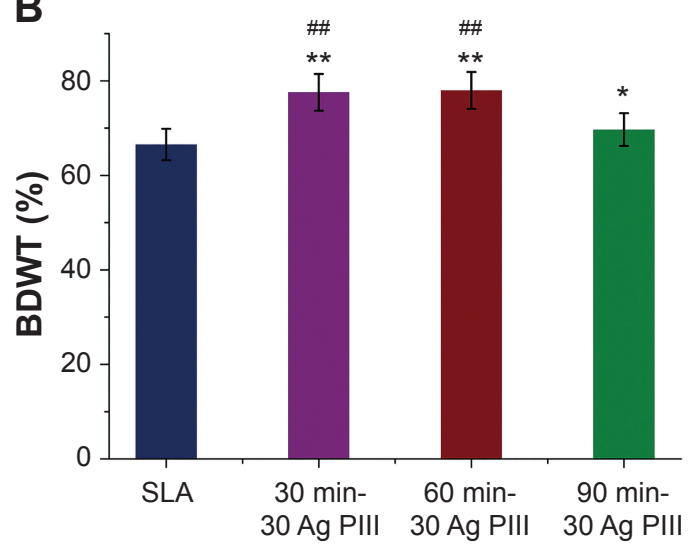

C

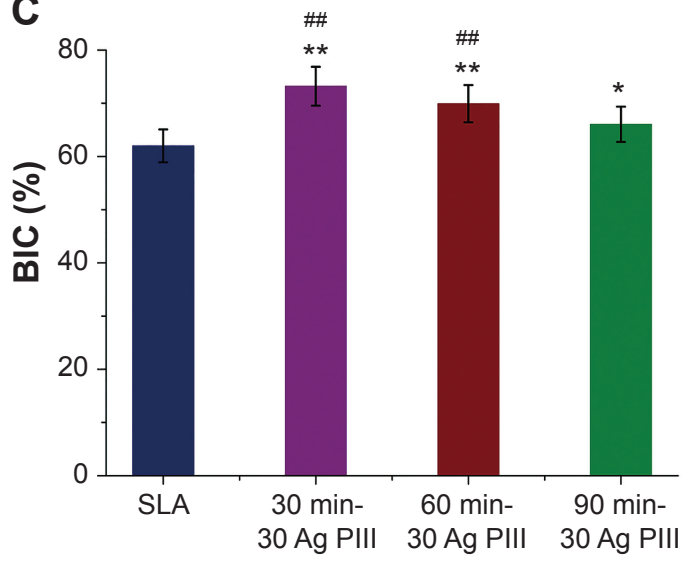

Figure 7 Histological observations and histomorphometric measurements.

Notes: Histological sections stained with van Gieson's picro fuchsin, from left to right, SLA group, 30 min-30 Ag PIII, 60 min-30 Ag PIII, 90 min-30 Ag PIII (A); The area of interest for the analysis of bone density within the threads ( $\mathbf{A}$, the green rectangle area); The BDWT and BIC of the bone volume adjacent to the implant surface were quantified (B and $\mathbf{C})$; ${ }^{* P}<0.0$ I versus the control SLA group; ${ }^{* * P}<0.05$ versus the control SLA group; ${ }^{\#} P<0.01$ versus the 90 min-30 Ag PIII group.

Abbreviations: Ag-PIII, silver plasma immersion ion implantation; BDWT, bone density within the threads; BIC, bone to implant contact; SLA, titanium surfaces treated by sandblasting with large grit and acid etching procedure; 30 min-30 Ag PIII, titanium surfaces treated by first SLA procedure and then silver plasma immersion ion implantation at $30 \mathrm{kV}$ for 30 minutes; 60 min-30 Ag PIII, titanium surfaces treated by first SLA procedure and then silver plasma immersion ion implantation at $30 \mathrm{kV}$ for 60 minutes; $90 \mathrm{~min}-30 \mathrm{Ag}$ PIII, titanium surfaces treated by first SLA procedure and then silver plasma immersion ion implantation at $30 \mathrm{kV}$ for 90 minutes.

effects. In the case of Ag NPs, the potential cytotoxicity effect becomes the biggest obstacle of application. Accordingly, in this study, for limiting silver uptake, the Ag-PIII technique was introduced to embed Ag NPs into titanium dental implants. Both clinical and histological measurements were applied to evaluate the osseointegration property of Ag-PIIItreated implants in vivo. Interestingly, no harm to the tissues adjacent to dental implants was observed, and even better osseointegration results were confirmed in Ag-PIII-treated groups compared with the SLA control.

Surface topographical characteristics of dental implants were earlier identified to be an important influencing factor of osseointegration. Bone tissues are hierarchical structures, which are composed of both micro- and nanoscale building blocks. From the biomimetic viewpoint, proper nanotopographically modified microrough surface may provide a more suitable surface for cell functions as it can better mimic the structure of the natural extracellular matrix. In previous studies, the cell abilities of adhesion, proliferation, and differentiation could be enhanced by nano/microtopographically modified titanium materials. These positive results were attributed to the combination effect of the nanoscale topography and the release of ions associated with bone osseointegration. ${ }^{47,48}$ In this research, the surface of the Ag-PIII groups is characterized by a hybrid micro/nanotopography, compared to the microrough structures of the SLA-surfaced implants. Although silver ions may be harmful to the cells and were not able to promote osseointegration, the histological observation found identical soft tissue attachments and enhanced new bone formation, BMD, and trabecular pattern. Considering the potential cytotoxicity effect of Ag NPs, mainly caused by common weakly bound particles, ICP was used to investigate silver release. Minimal silver release was confirmed, signifying 
minimal Ag NPs cytotoxicity. Actually, the bonding state of Ag NPs, obtained by the Ag-PIII technique, was quite different from other common techniques. The Ag NPs do not form a layer bound to the surface but become embedded in the titanium surface. In this case, the Ag NPs and the dental implants were completely composed together, so to avert cellular uptake and cytotoxicity. Therefore, the hierarchical structure combined with a low silver release property may constitute a convincing explanation for the better osseointegration results of Ag-PIII-treated groups.

SLA surface, a classic example of microrough surfaces, is still the most commonly used dental implant surface. This microrough surface can effectively promote osteoblast adhesion and differentiation, resulting in a faster osseointegration process. However, compared to a smooth surface, a smaller bone mass accumulation was observed due to the apparent depression of osteoblast proliferation. ${ }^{49}$ Nanotopographic modification can efficiently remedy for the defect. In the present study, we modified an SLA titanium dental implant surface with Ag NPs using the Ag-PIII technique. The Ag-PIII-treated groups exhibited prior osseointegration performance than the control. Moreover, Ag NPs of different sizes may perform differently. The 30 min-60 Ag PIII- and 60 min-30 Ag PIII-treated samples, with Ag NPs of size 5-25 nm, demonstrated superior bone apposition around implants to that seen for the 90 min-30 Ag PIII group, with Ag NPs of over $40 \mathrm{~nm}$. The difference may be related to the complex combination effect of Ag NPs and titanium (or titanium oxide) on physicochemical characteristics of the sample surfaces, which give rise to both controlled antibacterial activity and excellent proliferation of mammalian cells as we previously described. ${ }^{17,50}$ Therefore, it is reasonable to suggest that the size of particles may be a main contributing factor to the relatively inhibited bone apposition, emphasizing the importance of nanostructure in the osseoconductivity of biomedical implants. Ag NPs (5-25 nm) should be recommended for Ag-PIII-modified titanium surfaces. However, the actual mechanism still needs further clarification in future studies.

With respect to clinical evaluation, resonance frequency analysis was introduced as a noninvasive method whereby the formation of bone adjacent to an implant could be studied in vivo by measuring the resonance frequency of a small transducer connected to the implant fixture. ISQ, a unit for analyzing resonance frequency, is recorded as a number between 1 and 100, with 100 representing the highest degree of stability. Increased ISQ value is ascribed to increased bone anchorage, which is influenced by several factors, such as implant length and design, location of the first bone contact, degree of BIC, alveolar bone trabecular pattern, thickness of cortical bone, and bone density. ${ }^{39}$ Therefore, ISQ is a clinical tool to evaluate dental implant stability and reveals the condition of bone tissue formation around dental implants with limitation, especially at high ISQ levels $(>65)$. No correlations between histological parameters of osseointegration and ISQ values could be identified. Nevertheless, increased ISQ levels can still offer some hints on the state of osseointegration: implants with ISQ levels in the range from 57 to 82 are considered to be successfully integrated. ${ }^{51}$ In the present study, good primary stability (ISQ $>65$ ) was achieved at implant placement and decreased drastically during the first 4 weeks for all four groups of implants, with no significant differences, indicating baseline equivalency on dependent measures. The changing pattern of implant stability during the following 8 weeks was also similar in all the groups. However, the ISQ values were significantly higher for Ag-PIII groups compared to the control at 8 weeks, especially for $30 \mathrm{~min}-30 \mathrm{Ag}$ PIII and 60 min-30 Ag PIII groups. The gap between the groups shrunk in the last 4 weeks, but was still higher in the AgPIII groups. Considering the limitations of ISQ, it could be deduced that the rates of bone mineral apposition and speed of bone formation might significantly increase in the 30 min-30 Ag PIII and 60 min-30 Ag PIII groups. This was confirmed in our histological evaluation.

To summarize, this in vivo study demonstrated that hybrid micro/nanotopography played an important role in promoting bone apposition around endosseous dental implants, whereas the Ag NPs in the Ag-PIII-treated surface exhibited good biocompatibility for both the soft and hard tissue. Moreover, our data also emphasize the importance of the nanoparticle size in the osseoconductivity of implants. These results offer a potential use of Ag-PIII for antibacterial surfaces in the future. However, the definite relationship between nanoparticle size and new bone formation still needs to be investigated. In addition, it is important to point out that a very limited number of animals were used, and the antimicrobial properties of Ag-PIII-treated dental implants was not addressed in this animal study. Although being extensively studied in vitro, further work should include follow-up studies designed to evaluate whether the antimicrobial properties are retained in vivo and maintained in the long term.

\section{Conclusion}

In this study, different sized Ag NPs can be fabricated and embedded into the titanium dental implant surface using the Ag-PIII technique. Moreover, the silver release was minimized. 
The Ag-PIII samples exhibited outstanding compatibility to both the soft and hard tissue around dental implants. Enhanced bone formation was confirmed around the Ag-PIII-treated implant surfaces, which may be ascribed to the synergistic effects of the hierarchical hybrid micro/nanotopography. It is believed that the physical topography (micro- and nanoscale), which is closely related to the size and distribution of the fabricated Ag NPs, played a critical role in promoting osseoconductivity. These results suggest that Ag-PIII has the potential for future clinical use to modify implant surfaces.

\section{Acknowledgments}

Joint financial support from the National Basic Research Program of China (973 Program, 2012CB933600), National Natural Science Foundation of China (31370962, 81470782), Shanghai Committee of Science and Technology, People's Republic of China (13441902400, 14XD1403900) are acknowledged.

\section{Disclosure}

The authors report no conflicts of interest in this work.

\section{References}

1. Agarwal A, Weis TL, Schurr MJ, et al. Surfaces modified with nanometerthick silver-impregnated polymeric films that kill bacteria but support growth of mammalian cells. Biomaterials. 2010;31(4):680-690.

2. Martinez A, Guitián F, López-Píriz R, et al. Bone loss at implant with titanium abutments coated by soda lime glass containing silver nanoparticles: a histological study in the dog. PLoS One. 2014;9(1):e86926.

3. Zhao L, Wang H, Huo K, et al. Antibacterial nano-structured titania coating incorporated with silver nanoparticles. Biomaterials. 2011;32(24): 5706-5716.

4. Taheri S, Cavallaro A, Christo SN, etal. Substrate independent silvernanoparticle based antibacterial coatings. Biomaterials. 2014;35(16):4601-4609.

5. Reithofer MR, Lakshmanan A, Ping AT, Chin JM, Hauser CA. In situ synthesis of size-controlled, stable silver nanoparticles within ultrashort peptide hydrogels and their anti-bacterial properties. Biomaterials. 2014;35(26):7535-7542.

6. Martínez-Gutierrez F, Thi EP, Silverman JM, et al. Antibacterial activity, inflammatory response, coagulation and cytotoxicity effects of silver nanoparticles. Nanomedicine. 2012;8(3):328-336.

7. Castellano JJ, Shafii SM, Ko F, et al. Comparative evaluation of silver-containing antimicrobial dressings and drugs. Int Wound J. 2007; 4(2):114-122.

8. Furno F, Morley KS, Wong B, et al. Silver nanoparticles and polymeric medical devices: a new approach to prevention of infection? J Antimicrob Chemother. 2004;54(6):1019-1024.

9. Durán N, Marcato PD, De Souza GIH, Alves OL, Esposito E. Antibacterial effect of silver nanoparticles produced by fungal process on textile fabrics and their effluent treatment. J Biomed Nanotechnol. 2007;3(2):203-208.

10. Park EJ, Yi J, Kim Y, Choi K, Park K. Silver nanoparticles induce cytotoxicity by a Trojan-horse type mechanism. Toxicol In Vitro. 2010; 24(3):872-878.

11. Lubick N. Nanosilver toxicity: ions, nanoparticles - or both? Environ Sci Technol. 2008;42(23):8617.

12. Park MV, Neigh AM, Vermeulen JP, et al. The effect of particle size on the cytotoxicity, inflammation, developmental toxicity and genotoxicity of silver nanoparticles. Biomaterials. 2011;32(36):9810-9817.
13. Locht LJ, Smidt K, Rungby J, Stoltenberg M, Larsen A. Uptake of silver from metallic silver surfaces induces cell death and a pro-inflammatory response in cultured J774 macrophages. Histol Histopathol. 2011;26(6): 689-697.

14. Hussain SM, Hess KL, Gearhart JM, Geiss KT, Schlager JJ. In vitro toxicity of nanoparticles in BRL 3A rat liver cells. Toxicol In Vitro. 2005; 19(7):975-983.

15. Hussain SM, Javorina AK, Schrand AM, Duhart HM, Ali SF, Schlager JJ. The interaction of manganese nanoparticles with PC-12 cells induces dopamine depletion. Toxicol Sci. 2006;92(2): 456-463.

16. De Jong WH, Van Der Ven LT, Sleijffers A, et al. Systemic and immunotoxicity of silver nanoparticles in an intravenous 28 days repeated dose toxicity study in rats. Biomaterials. 2013;34(33): $8333-8343$.

17. Cao H, Liu X, Meng F, Chu PK. Biological actions of silver nanoparticles embedded in titanium controlled by micro-galvanic effects. Biomaterials. 2011;32(3):693-705.

18. Buser D, Mericske-Stern R, Bernard JP, et al. Long-term evaluation of non-submerged ITI implants. Part 1: 8-year life table analysis of a prospective multi-center study with 2359 implants. Clin Oral Implants Res. 1997;8(3):161-172.

19. Pikner SS, Gröndahl K, Jemt T, Friberg B. Marginal bone loss at implants: a retrospective, long-term follow-up of turned Brånemark System implants. Clin Implant Dent Relat Res. 2009;11(1): $11-23$.

20. Lekholm U, Gröndahl K, Jemt T. Outcome of oral implant treatment in partially edentulous jaws followed 20 years in clinical function. Clin Implant Dent Relat Res. 2006;8(4):178-186.

21. Massaro C, Rotolo P, De Riccardis F, et al. Comparative investigation of the surface properties of commercial titanium dental implants. Part I: chemical composition. J Mater Sci Mater Med. 2002;13(6): $535-548$.

22. Zinger O, Anselme K, Denzer A, et al. Time-dependent morphology and adhesion of osteoblastic cells on titanium model surfaces featuring scale-resolved topography. Biomaterials. 2004;25(14):2695-2711.

23. Cochran DL, Buser D, ten Bruggenkate CM, et al. The use of reduced healing times on ITI implants with a sandblasted and acid-etched (SLA) surface: early results from clinical trials on ITI SLA implants. Clin Oral Implants Res. 2002;13(2):144-153.

24. Jayaraman M, Meyer U, Bühner M, Joos U, Wiesmann HP. Influence of titanium surfaces on attachment of osteoblast-like cells in vitro. Biomaterials. 2004;25(4):625-631.

25. BuserD, Schenk RK, Steinemann S, FiorelliniJP, Fox CH, StichH. Influence of surface characteristics on bone integration of titanium implants. A histomorphometric study in miniature pigs. J Biomed Mater Res. 1991;25(7): 889-902.

26. Cochran DL, Schenk RK, Lussi A, Higginbottom FL, Buser D. Bone response to unloaded and loaded titanium implants with a sandblasted and acid-etched surface: a histometric study in the canine mandible. J Biomed Mater Res. 1998;40(1):1-11.

27. Buser D, Nydegger T, Oxland T, et al. Interface shear strength of titanium implants with a sandblasted and acid-etched surface: a biomechanical study in the maxilla of miniature pigs. J Biomed Mater Res. 1999;45(2):75-83.

28. Li D, Ferguson SJ, Beutler T, et al. Biomechanical comparison of the sandblasted and acid-etched and the machined and acid-etched titanium surface for dental implants. J Biomed Mater Res. 2002;60(2): $325-332$.

29. Lai HC, Zhuang LF, Liu X, Wieland M, Zhang ZY, Zhang ZY. The influence of surface energy on early adherent events of osteoblast on titanium substrates. J Biomed Mater Res A. 2010;93(1): 289-296.

30. Swart LC, Dreyer WP, van Zyl PP, Blignaut RJ. Early loading of mandibular implants placed immediately after extraction: a 10 -year prospective study of eight patients. Int J Oral Maxillofac Implants. 2014;29(6):1388-1396. 
31. van Velzen FJ, Ofec R, Schulten EA, Ten Bruggenkate CM. 10-year survival rate and the incidence of peri-implant disease of 374 titanium dental implants with a SLA surface: a prospective cohort study in 177 fully and partially edentulous patients. Clin Oral Implants Res. Epub 2014 Nov 05

32. Lai HC, Si MS, Zhuang LF, Shen H, Liu YL, Wismeijer D. Long-term outcomes of short dental implants supporting single crowns in posterior region: a clinical retrospective study of 5-10 years. Clin Oral Implants Res. 2013;24(2):230-237.

33. Papaspyridakos P, Chen CJ, Singh M, Weber HP, Gallucci GO. Success criteria in implant dentistry: a systematic review. J Dent Res. 2012;91(3): 242-248.

34. Costerton JW, Stewart PS, Greenberg EP. Bacterial biofilms: a common cause of persistent infections. Science. 1999;284(5418):1318-1322.

35. Glinel K, Thebault P, Humblot V, Pradier CM, Jouenne T. Antibacterial surfaces developed from bio-inspired approaches. Acta Biomater. 2012; 8(5):1670-1684.

36. Lee JH, Moon SK, Kim KM, Kim KN. Modification of TiO(2) nanotube surfaces by electro-spray deposition of amoxicillin combined with PLGA for bactericidal effects at surgical implantation sites. Acta Odontol Scand. 2013;71(1):168-174.

37. Nablo BJ, Rothrock AR, Schoenfisch MH. Nitric oxide-releasing solgels as antibacterial coatings for orthopedic implants. Biomaterials. 2005;26(8):917-924.

38. Pearce AI, Richards RG, Milz S, Schneider E, Pearce SG. Animal models for implant biomaterial research in bone: a review. Eur Cell Mater. 2007;13:1-10

39. Abrahamsson I, Linder E, Lang NP. Implant stability in relation to osseointegration: an experimental study in the Labrador dog. Clin Oral Implants Res. 2009;20(3):313-318.

40. Botticelli D, Persson LG, Lindhe J, Berglundh T. Bone tissue formation adjacent to implants placed in fresh extraction sockets: an experimental study in dogs. Clin Oral Implants Res. 2006;17(4):351-358.

41. Rossi F, Lang NP, De Santis E, Morelli F, Favero G, Botticelli D. Bonehealing pattern at the surface of titanium implants: an experimental study in the dog. Clin Oral Implants Res. 2014;25(1):124-131.
42. Sivolella S, Bressan E, Salata LA, Quiñones ME, Lang NP, Botticelli D. Deproteinized bovine bone mineral particles and osseointegration of implants without primary bone contact: an experimental study in dogs. Clin Oral Implants Res. 2014;25(3):296-303.

43. Vignoletti F, Abrahamsson I. Quality of reporting of experimental research in implant dentistry. Critical aspects in design, outcome assessment and model validation. J Clin Periodontol. 2012;39(Suppl 12):6-27.

44. Berglundh T, Stavropoulos A; Working Group 1 of the VIII European Workshop on Periodontology. Preclinical in vivo research in implant dentistry. Consensus of the eighth European workshop on periodontology. J Clin Periodontol. 2012;39(Suppl 12):1-5.

45. Zhang W, Wang G, Liu Y, et al. The synergistic effect of hierarchical micro/nano-topography and bioactive ions for enhanced osseointegration. Biomaterials. 2013;34(13):3184-3195.

46. Moulder JF, Stickle WF, Sobol PE, Bomben KD. Handbook of X-ray Photoelectron Spectroscopy. 2nd ed. Eden Prairie, MN: Perkin-Elmer Corporation, Physical Electronics Division; 1992.

47. Zhang W, Li Z, Huang Q, et al. Effects of a hybrid micro/nanorod topography-modified titanium implant on adhesion and osteogenic differentiation in rat bone marrow mesenchymal stem cells. Int $J$ Nanomedicine. 2013;8:257-265.

48. Zhang W, Li Z, Liu Y, et al. Biofunctionalization of a titanium surface with a nano-sawtooth structure regulates the behavior of rat bone marrow mesenchymal stem cells. Int J Nanomedicine. 2012;7: 4459-4472.

49. Zhao L, Mei S, Chu PK, Zhang Y, Wu Z. The influence of hierarchical hybrid micro/nano-textured titanium surface with titania nanotubes on osteoblast functions. Biomaterials. 2010;31(19):5072-5082.

50. Cao H, Qiao Y, Liu X, et al. Electron storage mediated dark antibacterial action of bound silver nanoparticles: smaller is not always better. Acta Biomater. 2013;9(2):5100-5110.

51. Ersanli S, Karabuda C, Beck F, Leblebicioglu B. Resonance frequency analysis of one-stage dental implant stability during the osseointegration period. J Periodontol. 2005;76(7):1066-1071.
International Journal of Nanomedicine

\section{Publish your work in this journal}

The International Journal of Nanomedicine is an international, peerreviewed journal focusing on the application of nanotechnology in diagnostics, therapeutics, and drug delivery systems throughout the biomedical field. This journal is indexed on PubMed Central, MedLine, CAS, SciSearch ${ }^{\circledR}$, Current Contents ${ }^{\circledR} /$ Clinical Medicine,

\section{Dovepress}

Journal Citation Reports/Science Edition, EMBase, Scopus and the Elsevier Bibliographic databases. The manuscript management system is completely online and includes a very quick and fair peer-review system, which is all easy to use. Visit http://www.dovepress.com/ testimonials.php to read real quotes from published authors. 